\title{
Learning Motivation and Its Related Factors in Chinese Classes in Senior High Schools
}

\author{
Junyi Zhang,
}

Beijing Royal School IB program, Beijing, 102218, China

E-mail:2148753991@qq.com

\begin{abstract}
Learning motivation is regarded as one of the important factors influencing learning performance and acquisition. A majority of the previous literature has focused on the strategies to improve students' learning motivation. However, less attention has been paid to investigate the underlying factors that affect students' learning motivation. Therefore, this study systematically reviewed the related studies. The results indicate that the extrinsic factors, namely, environmental factors, family factors and the teacher-student relationship can affect students' motivation in Chinese classes in senior high schools.
\end{abstract}

Keywords : Motivation, Chinese teaching, Extrinsic factors, Environmental factors, Family, Relationship

\section{INTRODUCTION}

The subject of Chinese or $\mathrm{Yu}$ Wen class plays a predominant role in senior high school. It is beyond a subject itself. For students in senior high schools, it is not only closely related to their current academic achievements but also has a great impact on their further education. To a certain extent, historical events and celebrity thoughts can help students learn how to think deeply and help students shape their outlook on life. More importantly, it is an indispensable method to pass down Chinese culture generation by generation. However, in the current high school teaching context, the course of Chinese has encountered lots of problems. Improving the teaching quality of Chinese is the most concerned issue for teachers. Different teaching strategy leads different motivation of study, and for improving students' motivation, it is necessary to suggest effective teaching methods, for which it is necessary to find out the factors that affect teaching effectiveness.

Among the related factors, motivation in learning is most frequently addressed. Learning motivation refers to an internal strength or internal mechanism that "promote, guide and maintain learning activities" [7]. Students' motivation is closely related to their learning performance and acquisition[2]. In addition, previous studies have stated a positive correlation between motivation and learning attitudes [3]. Students with strong motivation are more likely to have a positive attitude towards learning. When confronted with learning difficulties, they have strong perseverance and show high learning enthusiasm. Therefore, exploring the related factors that affect students' motivation and its application in instructions are of significant importance to improve students' performance and acquisition.

Therefore, by reviewing the previous literature, this study centers on the extrinsic factors that affect the motivations of high school students, intending to investigate the relationship between extrinsic motivation and teaching activities in the context of $\mathrm{Yu}$ Wen or Chinese class.

\section{DEFINITION AND TYPES OF STUDENTS' LEARNING MOTIVATION}

There is a consensus that motivation is "one of the important factors influencing the success of second language learning"[7]. However, with regards to its definition, there is no agreed definition among scholars. The most quoted definition in language learning is from Gardner [5], who defines it as the integration of efforts and desires to achieve the learning objectives and positive attitudes towards learning. According to the definition, it can be concluded that motivation is comprised of three components, namely, the efforts to achieve the objectives, the desire to achieve those goals and the positive attitudes towards language learning. In general, motivation refers to "a force that energizes, sustains, and directs behavior toward a goal"'[9]. Based 
on the definition, Gardner and Lambert [6] classify motivations into integrative motivation and instrumental motivation. Integrative motivation refers to the positive attitudes towards the target learning content, while instrumental motivation is the desire to learn.

However, the most influential distinction in motivation studies lies in the intrinsic and extrinsic distinction. Intrinsic motivation is involved in the learning process itself. It is about the basic psychological needs of the learners[10]. Intrinsic motivation is individually different. In instructional settings, intrinsic motivation mainly includes curiosity, thirst for knowledge, interest, and competition[1]. Extrinsic motivation is considered the productive force of intrinsic motivation. It refers to "the motivation to engage in an activity as a means to an end"[9]. A student with highly extrinsic motivation may study hard, in order to get rewards or avoid a failed score. Sometimes, extrinsic motivation is related to the learning process itself. It is involved in the factors that affect the studies. The distinguished extrinsic motivations are probably teachers' stimulation or incentives and peer recognition and admiration[1]. For students in senior middle schools, their learning motivation is affected by intrinsic and extrinsic motivation, while extrinsic motivation has a greater impact on their studies[7].

\section{Descriptive Analysis of the Previous Literature}

This study searched the related literature in CNKI (China National Knowledge Infrastructure), the biggest literature database. It is an important database to collect the published journals, dissertations, proceedings, books, and even newspapers. When searching the keywords "Chinese teaching in senior high school", people can find 43,463 related results. The studies on Chinese teaching are a flourishing and productive field. As Figure 1 shows, the research on Chinese teaching in high schools can date back to the 1980s. There has been a dramatic surge over the past two decades. It indicates that Chinese teaching becomes increasingly important in middle school teaching activities.

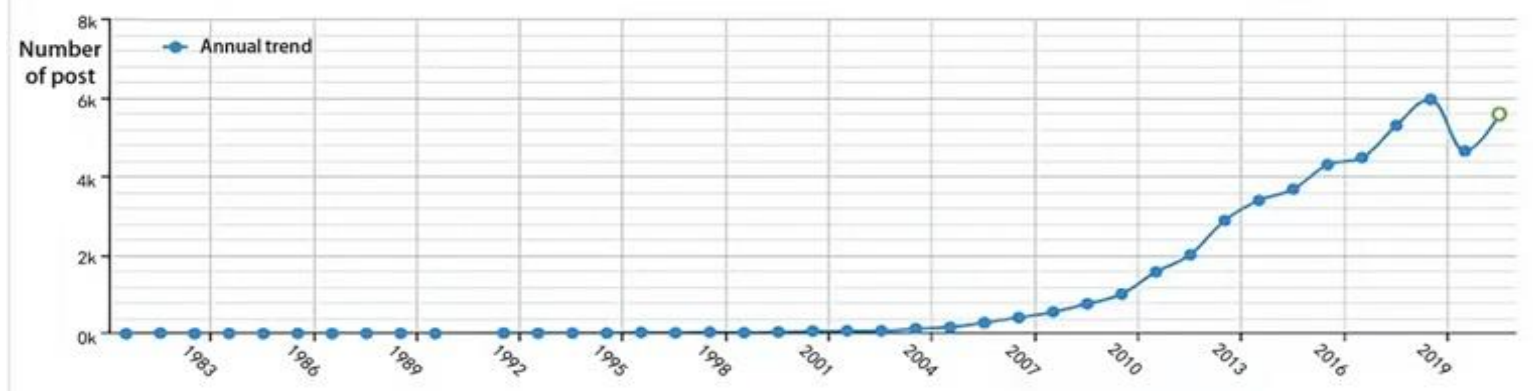

Figure 1 The trend of published articles about Chinese teaching in senior high school

However, when searching the keywords "Chinese teaching in senior high school" and "motivation", there are only 293 related studies in total. Figure 2 displays the trend of published articles about students' motivation in Chinese classes in senior high school. The earliest study was published in 2009, which is a thesis about the influence of teachers' language on teaching performance and students' acquisition. Among those related articles, most studies focus on the employment of new teaching methods in Chinese classes and the strategies for improving student's motivation in learning Chinese. Less attention has been paid to explore the related factors impacting students' motivations. A majority of studies point out a common phenomenon, which is the low motivation of students in Chinese classes. Therefore, how to improve students' motivation becomes a mainstream of studies. However, this study attempts to investigate the factors that affect students' motivations. Understanding the factors at work can contribute to the improvement of students' motivations. 


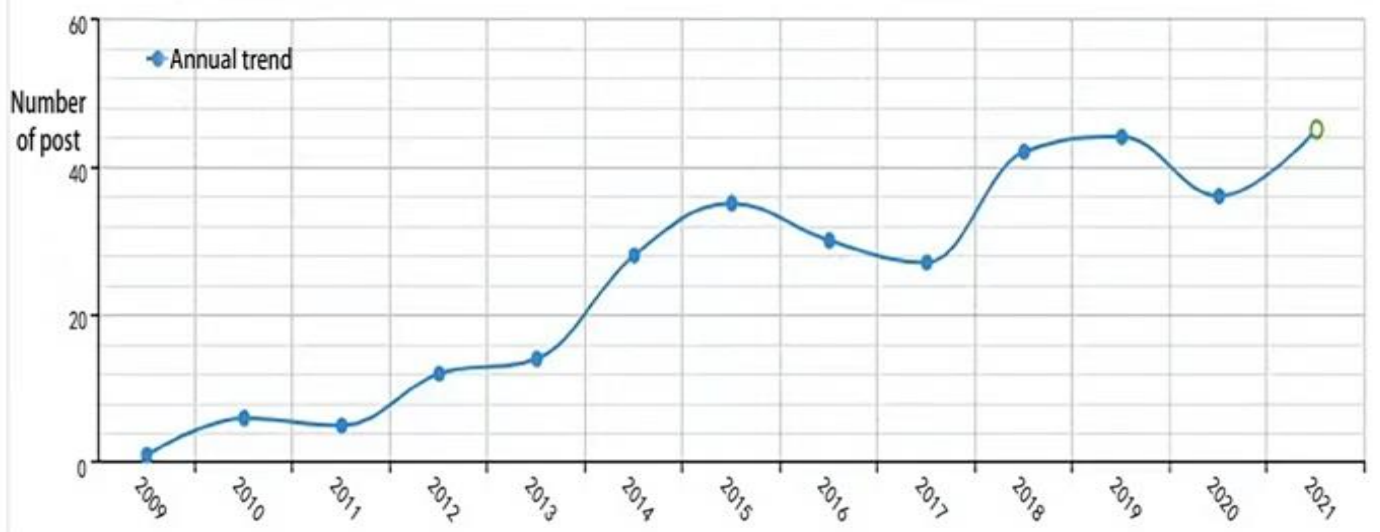

Figure 2 The trend of published articles about students' motivation in Chinese classes in senior high school

\section{EXTRINSIC FACTORS AFFECTING STUDENTS' MOTIVATIONS}

Through a systematic literature review, this study summarized three significant extrinsic factors that influence students' motivations, namely, the environmental factors, family factors, and the relationship between teachers and students.

\subsection{Environmental Factor}

In a study on the students' learning motivation, Fang [4] displayed the Two Factor Theory proposed by Herzberg to investigate the influence of environmental factors. Two Factor Theory, or Herzberg's motivation theory model, was initially developed in 1959 and designed to explain the root of motivation in the workplace[8]. In this model, Herzberg identified two significant factors, including motivating factors and hygiene factors. The motivating factors are related to the job it self, while the hygiene factors are the factors surrounding the job. Based on Herzberg's two-factor theory, Fang claims that in instructional settings, the hygiene factors are mainly the school's living facilities, teaching facilities, and campus environment, while the motivating factors refer to students' life value, a cognitive system, professional prospects, and among others. Hygiene factors affect students' learning motivation through management level, supervision level, relationship with teachers, learning conditions, safety and so on. This factor can also eliminate or increase student dissatisfaction. Motivating factors affect students' learning motivation by showing the different extent of sense of accomplishment, recognition of studying performance, degree of difficulty, opportunities for future life, etc., which can prompt or undermine students' enthusiasm for learning. These two factors eventually influence students' academic performance.

Fang also stated that the hygiene factors are related to extrinsic motivations. It mainly refers to the related learning environmental factors, including campus environment factors, social environment, and family environment. These factors only meet the low-level physiological needs and safety needs of students.

\subsubsection{The neighborhood environment of the school}

The surrounding environment of the campus refers to the environment where the campus is located. Creating a good surrounding environment is not only the need of the city's development but also an important task to promote the healthy growth of young people. The pleasant neighborhood environment, the healthy cultural and spiritual atmosphere, and the diversified extracurricular activities can promote the extrinsic motivation of students.

\subsubsection{Campus environment}

The campus environment is an explicit culture of school culture. It includes the constructions of the school, the teaching facilities, the students' living facilities, and extracurricular activities. The quality of these material and cultural constructions is closely related to the improvement of students' learning motivations. A good learning environment and living atmosphere will undoubtedly inspire people's spirits and generate positive intrinsic motivations for learning. All of these will drive students consciously to pursue progress and work hard. Various extracurricular activities would enrich students' life and help them realize the value of knowledge. In addition, harmonious interpersonal relationship is part of the campus environment. Building a harmonious interpersonal relationship in a school is mainly based on equality, respect, mutual understanding, and tolerance among teachers and students. A harmonious relationship can also improve students' learning motivations.

It is worth mentioning that environmental factors mainly influence the students' extrinsic motivations. 
Even though, they are, no doubt, an indispensable part of teaching settings. First and foremost, parents are required to create a learning environment for children. every family member is actively involved in learning activities and provides an array of learning materials. Children can learn Chinese knowledge unconsciously.

\subsection{Family factor}

A family is a small unit in society. It serves as not only fertility, economic functions, rest, or entertainment, but also functions as education. The educational function of families is closely related to the family environment. The family environment includes an array of factors, for example, the economic status, parents' educational backgrounds, and educational methods. In our country, parents are obliged to bring up children and educate them. Due to the special status of families, families play an indispensable part in education, which is impossibly replaced by schools and other institutions. Children or adolescents who have lost family education and poor family education are prone to encounter difficulties in the process of their socialization, leading to certain negative problems.

In a study to explore the factors that affect students' learning motivations, Reheman (2017) reveals the negative influence of single-parent families on students' learning. Good family education is more likely to lead to good study habits of students. However, the increasing divorce rate results in growing single-parent families. The incomplete family is prone to a lack of supervisory. The limited energy of single parents would probably lead to unsupervised children in learning. In addition, the bad relationship between parents would have a negative influence on the development of children's personalities and studying habits. These students tend to be confronted with problems in learning motivations and learning habits.

Family education plays a more significant role in Chinese learning than that in other subjects. Family education is the starting point of children's education. Parents are the first teacher of children. High-quality family education can provide students with a good environment in learning language and literature. Wang[11] offered suggestions on the role of family education in students' motivation in learning Chinese. Next, the participation of parents in Chinese education would facilitate children's learning. The involvement of parents has a positive impact on improving students' interests and learning motivations. Finally, parents should help students develop a reading habit. Reading is an important habit for Chinese learning and has a significant role in acquisition and performance. Parents could have more opportunities to cultivate students' reading habits.

Based on the discussions above, family plays a crucial role in students' learning. The quality of family education would affect students' thinking and behaviors. Eventually, they would influence students' learning motivation.

\subsection{The relationship between teachers and students}

The relationship between teachers and students is the most basic interpersonal relationship in education. A good teacher-student relationship can not only promote the development of education and teaching activities but also contribute to students' physical and mental health. However, for a long time, the teacher-student relationship in Chinese teaching in senior high schools has encountered a problem of alienation and utilitarianism. Therefore, in order to improve the efficiency of Chinese teaching in high schools, it is necessary to build a harmonious teacher-student relationship. In the traditional Chinese culture, teachers often take a dominant position in class, while students tend to be passive recipients. However, in recent years, the relationship between teachers and students has experienced significant changes. Their relationship grows more equal in class. The teacher-oriented class has shifted into a learner-oriented class.

Xiao [12] indicated the positive correlation between teacher-student relationships and students' motivation. A harmonious teacher-student relationship can improve students' learning motivation. Previous studies prove that students' learning is susceptible to the influence of teachers' teaching styles. If students like the teaching style, they are more likely to actively engage in the class. Therefore, the harmonious relationship between teachers and students can greatly improve students' learning enthusiasm and initiative. Likewise, teachers also need students' acceptance and recognition. Students' love and acceptance would encourage the teachers and facilitate the teaching process. This would result in a virtuous circle.

Given this, Zeng [13] made advice building a harmonious teacher-student relationship in her study. First of all, learn to respect students. Respect is a prerequisite for education. Respect is the most fundamental aspect of education. Second, love your students. Love is the nature of education. Devotion is the guarantee of education. Without teachers' love, it is impossible to make students enjoy the learning process. Thirdly, understand the students. Teachers should have a deep understanding of each student's learning situation, understand their learning level, set different goals for them, and strive to become their friends. More importantly, teachers need to create a relaxed teaching environment. A relaxed situation can stimulate students thinking and create good conditions for students. Finally, use different teaching methods. Different teaching methods can promote the teaching process and 
attract students' interest in learning. All of these are good attempts to establish a harmonious teacher-student relationship.

\section{CONCLUSION}

Learning motivations in Chinese teaching is not a new topic in educational studies. Over the decades, it has received increasing attention from scholars. However, less attention has been paid to investigate the factors that affect students' motivation. This study primarily focused on the extrinsic factors that influence students' learning motivations in the class of Chinese in high schools, to identify the underlying relationship between motivations and Chinese learning. By reviewing the previous literature, three significant extrinsic factors have been identified. The environmental factors, family factors, and teacher-student relationships are closely related to extrinsic motivations. They play a significant role in affecting students' motivation. In the future, more in-depth and empirical studies on the underlying factors are needed.

\section{ACKNOWLEDGMENT}

Firstly, I would like to show my deepest gratitude to my teachers and classmate in my highschool, who have provided me with valuable guidance in every stage of the writing of this thesis. Secondly, I would like to thank all my family members for their encouragement and support. Further, I would like to appreciate my friends who persuaded me to write this thesis. Without all their enlightening instruction and impressive kindness, I could not have completed my thesis.

\section{REFERENCES}

[1] An, M. (2010). On Training and Stimulate Students Learning Motivation. Modern Reading.

[2] Anderman, L. H., \& Midgley, C. (1998). Motivation and middle school students. Perspective, 26(3/4), 325-346.

[3] Ausubel, D. A. (1968). Educational Psychology: A Cognitive View. New York: Holt, Rinehart \& Winston.

[4] Fang, X. (2013). A study of the effect of two factor theory on the learning motivations of middle school students. Youth, 327-328.

[5] Gardner, R. (1985). Social Psychology and Second Language Learning: The Role of Attitude and Motivation. London: Edward Arnold.

[6] Gardner, R., \& W. Lambert. (1959). Motivational variables in second language acquisition . Canadian Journal of Psychology.
[7] Long, C., Ming, Z., \& Chen, L. (2013). The Study of Student Motivation on English Learning in Junior Middle School--A Case Study of No. 5 Middle School in Gejiu. English Language Teaching, 6(9), 136-145.

[8] Ozguner, Z., \& Ozguner, M. (2014). A managerial point of view on the relationship between of Maslow's hierarchy of needs and Herzberg's dual factor theory. International Journal of Business and Social Science, 5(7), 207-215.

[9] Paul, E., \& Kauchak, D. (2005). Educational Psychology: Windows on Classroom. Shangxi: Shangxi Normal University Press.

[10] Van Lier, L. (1996). Interaction in the language curriculum: Awareness, Autonomy and Authenticity. London: Longman.

[11] Wang, G. Z. (2019). Research on Strategies of Family Education to Improve Students' Interest in Chinese Learning. Gao Kao, 89.

[12] Xiao, Y. S. (2016). Analysis of the role of teacher-student relationship in Chinese teaching in senior high schools. Hua Xia Jiao Shi, 10.

[13] Zeng, Y. (2007). The Construction of Harmonious Teacher-student Relationship in Chinese Teaching. Sichuan University of Arts and Science Journal, (17), 59-60. 